\title{
System Identification and Model Predictive Control using CVXGEN for Electro-Hydraulic Actuator
}

\author{
Xuan Wong Liang', Ahmad 'Athif Mohd Faudzi'", Zool Hilmi Ismail' \\ ${ }^{1}$ Malaysia-Japan International Institute of Technology, Universiti Teknologi Malaysia, Jalan Sultan Yahya Petra,54100 \\ Kuala Lumpur, Malaysia \\ ${ }^{2}$ School of Electrical Engineering, Faculty of Engineering, University Teknologi Malaysia, 81310 Johor Bahru, Johor, \\ Malaysia
}

${ }^{3}$ Centre for Artificial Intelligence and Robotics, Universiti Teknologi Malaysia, Jalan Sultan Yahya Petra,54100 Kuala Lumpur, Malaysia

*Corresponding Author

DOI: https://doi.org/10.30880/ijie.2019.11.04.018

Received 25 April 2019; Accepted 1 August 2019; Available online 5 September 2019

\begin{abstract}
Hydraulics have been widely used in heavy industries for decades. The demand for intelligent hydraulic control system has been increasing as tough robotic researches are getting more popular. Despite the high power to weight ratio delivery, the hydraulic actuator suffers from nonlinearity properties that cause difficulties in applying precise position control. In this paper we proposed Model Predictive Control (MPC) to control an ElectroHydraulic Actuator (EHA) where its dynamic characteristics is obtained through system identification method. Control signal generation optimisation and constraint handling are seldom included in the conventional control system design process. Therefore we introduce CVXGEN, a Code Generator for Embedded Convex Optimization that utilises the Quadratic Programming (QP) interior-point solver for MPC optimisation problem. Predictive Functional Control (PFC) is used to validate the CVXGEN-MPC and both algorithms are implemented in simulation and experiment of EHA position control to highlight the optimisation and constraint handling problem. Control performance, control effort, constraint handling and disturbance handling of both methods are discussed.
\end{abstract}

Keywords: electro-hydraulic actuator, system identification, model predictive control, optimisation.

\section{Introduction}

\subsection{Hydraulic Actuator}

The hydraulic system has been widely applied in heavy industries for many decades. It is majorly used in heavyduty machineries for example, power steering system [1], excavator [2], rough grinding machine [3] and tractor [4]. The fluid power system can withstand sudden overload and stall without damage. Furthermore, a low compression ratio of fluid allows the power to be transmitted almost instantaneously. Last but not least, the power per weight ratio of hydraulic system is better when compared to conventional system [5], [6].

Commercial heavy-duty machinery uses oil in hydraulic system because of the special characteristics of oil. Oil has an excellent performance on heat dissipation and oil also act as lubricant in the power system. Nonetheless, hydraulics system also showed great result when compared with electric system. The torque of an electric motor is proportional to current supplied and it is limited by magnetic saturation while the hydraulic motor is depended by pressure difference provided by pump. The hydraulic system also has a higher or faster response to change in speed or direction than electric system [7]. 
However, despite many advantages of fluid power system, there are also some unavoidable drawbacks. On the other hand, leakage happens if the components or parts of hydraulic system were not designed and fabricated properly. Fluid power system requires a pump motor and the system often takes a large space to setup [5]. Fluids are known with non-linear physical properties [8]. Thus, the hydraulic system is also a non-linear system and it is difficult to obtain its mathematical model. The nonlinearities of hydraulic are mainly caused by the fluid characteristics, friction of piston in chamber as well as internal and external fluid leakage. Moreover, intelligent control systems are required for hydraulics system to increase the efficiency. Therefore, many studies have been carried out by researchers to overcome such existing problems of hydraulic system [9]-[12].

\subsection{Control Algorithms}

Intelligent controllers for tough hydraulics systems are essentials to improve the efficiency while maintaining the control performance. Nadeau, Micheau and Boisvert have proposed to apply model-based predictive control to a dual regenerative and hydraulic brake system of a three-wheel electric vehicle in simulation [13]. However, they claim to face issues with real-time model predictive control in the control system due to online identification and controller robustness. Tomatsu et al. have implemented model predictive trajectory tracking control on hydraulic excavator for digging operation [14]. The effectiveness of combining hydraulic servo mechanism and predictive control has been proven even with the existence of disturbance. Chihi et al. have published an intelligent Proportional-Integrative (PI) controller for the electro-hydraulic system [15]. The method utilises model-free controller and combine with conventional PID controller and simulated with an electro-hydraulic system. The method is easy to implement to a complex system with the non-linear characteristic. A fault-tolerant controller system has been developed by Sun and his research members [16]. They have designed a multi-level model predictive control for detection filter and backup control. The intelligent control system can detect actuator faults and switch immediately to the backup control.

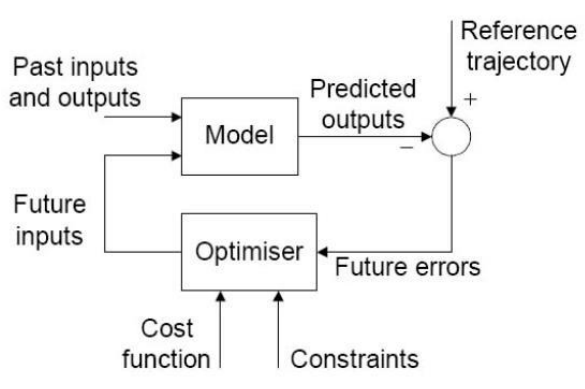

Fig. 1 - Basic Structure of MPC.

Control engineers utilise the mathematical model of plant to design a control system suitable for a specific purpose. Therefore, it is essential to obtain the model of control plant before design the control system. Control plant model identification is aided by modern computing software nowadays such as Matlab. System identification relates the input and output of system and determines the model of control plant in mathematical formula. Model Predictive Control (MPC) utilises the approximation model of control plant to predict and produce the best control input for the plant. MPC has been implemented into a vast area of industries including automotive, chemical and food processing. The basic structure of MPC is shown in Figure 1. There are several algorithms which branched out from MPC such as Dynamic Matrix Control (DMC) [17], Model Algorithm Control (MAC) [18], Extended Horizon Adaptive Control (EHAC), Generalised Predictive Control (GPC) [19], Modified Predictive Control [20] and Predictive Functional Control (PFC) [21].

MPC can handle constraints, robust and multivariable control when compared with widely-accepted conventional PID controller. Furthermore, a constraint of a dynamic actuator is able to be included in the MPC to improve the power efficiency [22]. These are the important considerations which a PID controller would not able to handle. However, the implementation of optimisation problem of MPC with constraints, the process is complicated and challenging. The optimisation is considered as a Quadratic Programming (QP) problem. A general solver might be able to solve different QP problems however its pre-built library is often bigger in memory size and slower in online computation. The QP problem solver is required to be robust, fast and simple footprint [23]. Code generation for specific QP problem solver can shorten the processing time on finding optimised control input and also reduce the data size of the solver. Therefore, CVXGEN, the automatic code generation for embedded convex optimization introduced by J. Mattingley [24] is a powerful tool in helping to solve the QP problem effectively. 


\subsection{Control Strategy}

Predictive Functional Control (PFC) algorithm and Model Predictive Control (MPC) implemented in CVXGEN algorithm are applied to EHA to compare and discuss the performances in simulation and experiment respectively. CVXGEN is a greatly optimised Quadratic Programming (QP) solver for MPC, while PFC is derived intuitively from MPC theories [25] and it is fairly easy to deploy into control system.

Predictive Functional Control is first developed by Richalet [25]-[27] in 1968. PFC is developed to overcome the high online computation effort of MPC, which can be only applied on long sampling time control system, chemical plant for instance. PFC has a basic control theory and therefore greatly reduce the computational effort of a control system. Since then, application of PFC has been widened into military [22] and robotics [28].

The control theory of PFC rather simple and straight forward. It employs the state-space model of actuator and predicts the future output given a virtual input. State-space prediction model can easily handle systems with non-zero initial conditions and multi-variable system [29], [30]. PFC is composed of three main components which are prediction mode, reference trajectory, and control law.

A state space discrete model given is used. This process is simply an iteration of an n-steps ahead prediction and repeated substitution results can be generalised into the following equation. The state space model of $n$-step ahead prediction can be formulated and shown in Equation 1 and Equation 2.

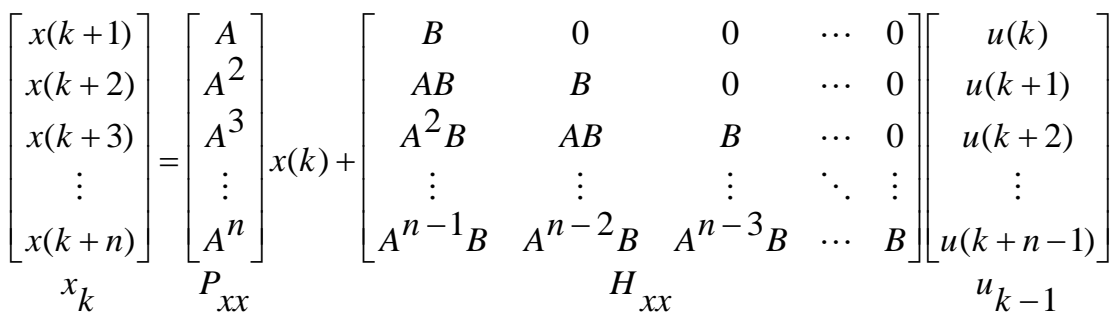

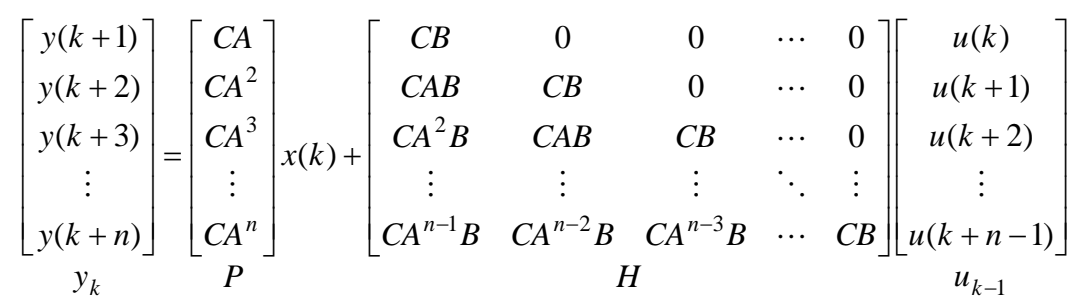

where $P_{x x}, H_{x x}, P$ and $H$ are matrices and vectors of the right dimension. Thus, the state model, $x_{k}$ and measured output, $y_{k}$ are defined as as shown in Equation 3 and Equation 4.

$$
\begin{gathered}
x_{k+1}=P_{x x} x_{k}+H_{x x} u_{k} \\
y_{k}=P x_{k}+H u_{k-1}
\end{gathered}
$$

Reference trajectory is given to system to mimic the behaviour of first order system with delay [31]. Therefore, the desired closed-loop dynamic an in the reference trajectory formula. The one step ahead reference point, $\omega$ is shown in Equation 5 and Equation 6..

$$
\begin{gathered}
\omega_{k+1}=r_{k}-\left(r_{k}-y_{k}\right) \psi^{i} \\
\psi=e^{-T_{s} / \tau_{d}}
\end{gathered}
$$

where $r$ is the set point, $y$ is the feedback, $\psi^{i}$ is the tuning parameter which its value ranges from zero to one, while $n$, $T_{s}$ and $T_{d}$ is prediction step, sampling time and time constant for first-order system response respectively. 
In order to acquire the control input, $u_{k}$ at $k$ instance, the equation is rearranged and with the assumption $u_{k-1}=u_{k}$, since only the first control input will be applied to the control system, control input, can be obtained by using Equation 7.

$$
u_{k}=H^{-1}\left(-P x_{k}+r_{k}-\left(r_{k}-y_{k}\right) \psi^{i}\right)
$$

A state estimator is employed in the algorithm to estimate the state variables based on the feedback from the actuator [21], [28].

CVXGEN is introduced by J. Mattingley and Boyd in 2011 as a convex optimisation solver. It fulfils the requirements making embedded optimisation possible [32]. First and foremost, the user has to declare the QP problem in CVXGEN specification language. CVXGEN then will translate the QP problem and generate lightweight custom C solver. Given its fast and small code size, the user can apply the solver in various kind of embedded system as CVXGEN targets small-sized problems [23], [32], [33].

Consequently, the QP problem needs to be specified in CVXGEN. Dimensions, variables and parameters are needed to state clearly in CVXGEN. The control objective of MPC is to control the actuator so it tracks the planned trajectory and minimises the changes of control input. The cost function in Equation 8 shows the control objective.

$$
v(k)=\sum_{i=H_{w}}^{H_{p}}\|\widehat{z}(k+i)-r(k+i)\|_{Q(i)}^{2}+\sum_{i=0}^{H_{u-1}}\|\Delta \widehat{u}(k+i)\|_{R(i)}^{2}
$$

where $\hat{z}$ is the predicted output, $r$ is the reference trajectory, $\Delta \hat{u}$ is the change of control input, $Q$ and $R$ are diagonal weighting matrix, $H_{p}$ is the prediction horizon, $H_{w}$ is window parameter, $H_{u}$ is control horizon, $k$ is the number of sample in discrete time system. Idealistic control system has zero lag when responding to input while such condition does not happen in actual control system. Hence, window parameter is introduced to start predict the feedback of actuator after a few sampling time later to improve the accuracy of control system. While in the control horizon, cost function calculates and apply the change of control input.

The QP problem must be identified and rearrange into the canonical form before any further steps can take place [34]. CVXGEN will use a standard primal-dual interior point method to find the solution for the problem. The primaldual interior point method is appropriate to be installed into embedded system and reliable to find a solution with high accuracy. The canonical form of a general QP problem is shown in Equation 9.

$$
\begin{array}{cc}
\text { minimise } & \frac{1}{2} x^{T} Q x+q^{T} x \\
\text { subject to } & G x \geq h, A x=b
\end{array}
$$

\section{Methodology}

The following section is presented by setup of the experiment, followed by the model identification using system identification toolbox in Matlab. Consequently after the model of EHA is acquired, both simulation and experiment of EHA position control will be performed based the model obtained earlier. MPC is represented in CVXGEN will be implemented in the simulation and experiment, where the result will be collected and validated by PFC. Nevertheless, the same setup is then rerun to test for the toughness of the control algorithms verify for their disturbance compatibility.

The experiment is run in Matlab Simulink with Real-time Windows Target as solver, using National Instrument PCI-6221 data acquisition card. The experiment is run in Matlab Simulink with Real-time Windows Target as solver, using National Instrument PCI-6221 data acquisition card. Figure 2 shows the experiment setup in this study. 


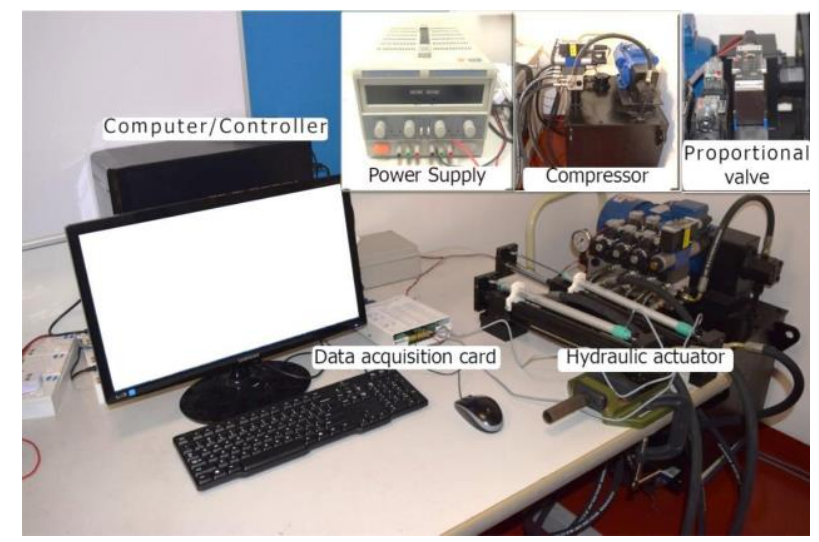

Fig. 2 Interface between hydraulic and computer system.

The proportional valve used in the experiment is Bosch Rexroth 4WREE6 and it operates at 24V DC. It allows a nominal flow rate of 8 litres per min and its maximum operating pressure is at 315 bar. The control signal of the valve is $\pm 10 \mathrm{~V}$. On the other hand, the hydraulic cylinder has a bore of $30 \mathrm{~mm}$, which has a stroke of $250 \mathrm{~mm}$. Its maximum working pressure is 230 bar and maximum flow rate is 8 litres per minute. The displacement sensor used in this experiment is PZ-34-A-250, which is manufactured by Gefran Group. It is a linear potentiometer with an infinite resolution.

The hydraulic system and computer where the controller located were interfaced by a data acquisition system (DAQ). DAQ system connects the hardware and the computer by converting sensor data into interpretable signals for the computer to process it. A National Instruments product, PCI-6221 DAQ system is used in this experiment. It has a maximum sampling rate of $250 \mathrm{kHz}$. It supports up to 16 analogue inputs and outputs, and 24 digital inputs and outputs.

A simulation and experiment are run with the method PFC, by Izzuddin and Osman [29], [37] and CVXGEN-MPC [31], based on the 3rd order state space model while the model of electro-hydraulic actuator (EHA) is obtained by using the system identification toolbox in Matlab. The Auto-Regressive with Exogenous Input (ARX) model is used in the experiment to obtain the model of EHA. The model of EHA acquired in the system identification experiment is $H(z)=\frac{B(z)}{A(z)}$, which is displayed in Equation 10.

$$
\begin{aligned}
& A(z)=1-2.106 z^{-1}-1.405 z^{-2}-0.299 z^{-3} \\
& B(z)=0.0044 z^{-1}+0.0224 z^{-2}+0.0283 z^{-3}
\end{aligned}
$$

The simulation and experiment are based on the settings shown in the Table 1. The prediction horizon is 20 and window parameter is set to 5, hence prediction horizon is range from 6th until 20th sample. Two constraints are added for CVXGEN-MPC, which are actuator slew rate and maximum input for the hydraulic valve.

Table 1 - Settings of simulation and experiment.

\begin{tabular}{cc}
\hline Settings & Values \\
\hline Sampling time & $0.01 \mathrm{~s}$ \\
Prediction horizon & 6th -20 th sample \\
Control horizon & 0th -4 th sample \\
Slew rate & $0.175 \mathrm{~ms} \neg-1$ \\
Allowable control signal & $\pm 10 \mathrm{~V}$ \\
Tuning parameter & $\tau \mathrm{d}=0.01$ \\
\hline
\end{tabular}

The implementation of CVXGEN-MPC and PFC approaches are implemented on EHA and carried out in simulation and experiment. Hence, the objective of simulation and experiment is to find out the effectiveness of optimisation given operational boundaries when CVXGEN-MPC and PFC are applied in position control of EHA. The control performance, signals and effort results are recorded and compared. Figure 8 shows the control performance of CVXGEN-MPC and PFC in simulation and experiment. 


\section{Results and Discussions}

Simulation and experiment results are often different in scale. This is probably due to inaccuracy of plant model and idealistic parameters in simulation or even unwanted noises when collecting data in experiment. The results in simulation and experiment with CVXGEN-MPC and PFC are tabulated and compared side by side to highlight the differences.

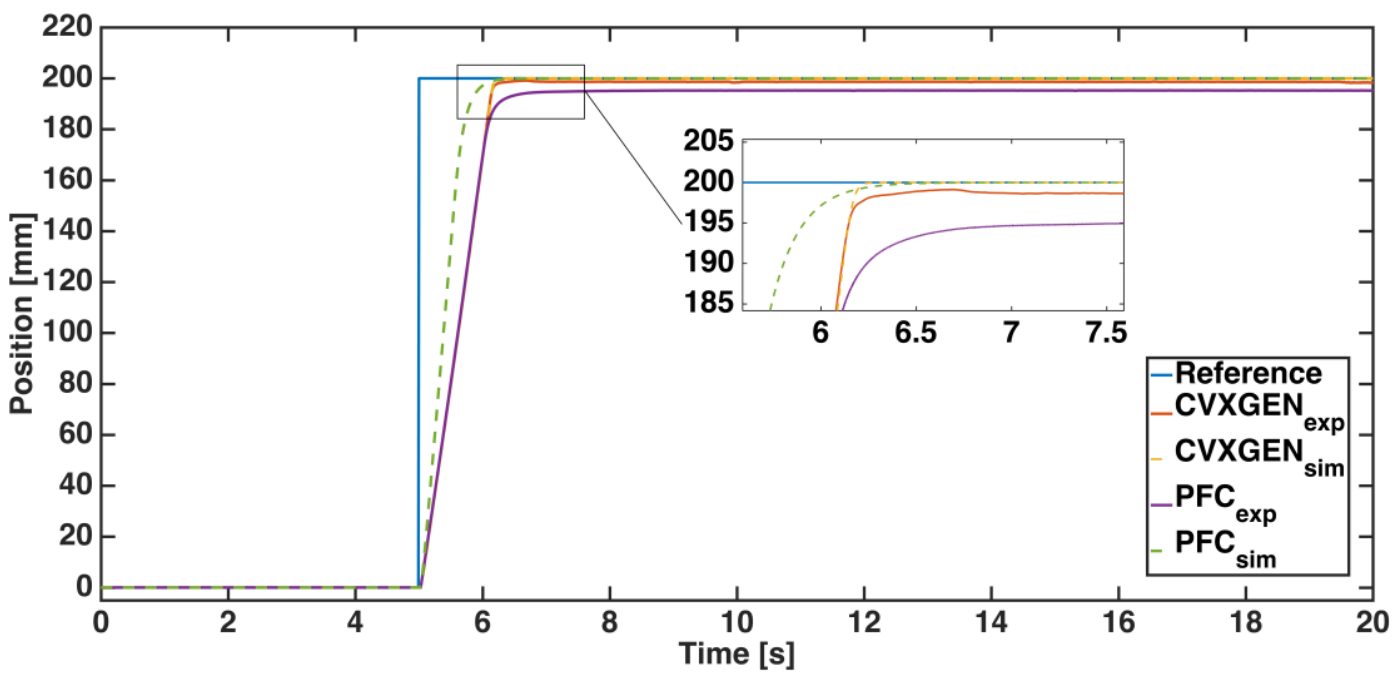

Fig. 3 Control response of CVXGEN-MPC and PFC in simulation and experiment.

In Figure 3, CVXGEN-MPC method shows a small difference between experiment and simulation results. On the other hand, experiment dynamic actuation feedback using PFC results in deviation in simulation output. This is due to lack of hardware limitation configuration in PFC, for instance, slew rate of actuation. Nonlinear feedback from CVXGEN-MPC output in the experiment is probably due to constraint is given to controller. Friction and unwanted noise are very likely to be the reason why steady state error exists in both experiment results.

Table 2 - Experiment result comparison.

\begin{tabular}{ccccc}
\hline Performance & \multicolumn{2}{c}{ CVXGEN-MPC } & \multicolumn{2}{c}{ PFC } \\
Specifications & Experiment & Simulation & Experiment & Simulation \\
\cline { 2 - 5 } Rise Time, Tr (s) & 0.903 & 0.911 & 0.895 & 0.587 \\
Settling Time, Ts (s) & 0.614 & 0.615 & 0.630 & 0.595 \\
Steady State Error (\%) & 0.430 & 0 & 2.347 & 0 \\
Normalised Mean-Square & \multicolumn{2}{c}{98.685} & & \multicolumn{2}{c}{87.564} \\
Error, NMSE (\%) & \multicolumn{2}{c}{} \\
\hline
\end{tabular}

Based on Table 2, the control performance of CVXGEN-MPC outperform PFC in the experiment results. The result CVXGEN-MPC shows a $0.9 \%$ longer in rise time, $2.5 \%$ shorter in settling time and $83.0 \%$ lesser in steady state error than the result of PFC. The normalised mean square error is determined to find the likeness between experiment and simulation result for both algorithms. With the simulation result as the reference, the experiment result of CVXGEN-MPC shows a $98.7 \%$ of resemblance with its simulation result, while there is only $87.6 \%$ of similarity between experiment and simulation result for PFC. 

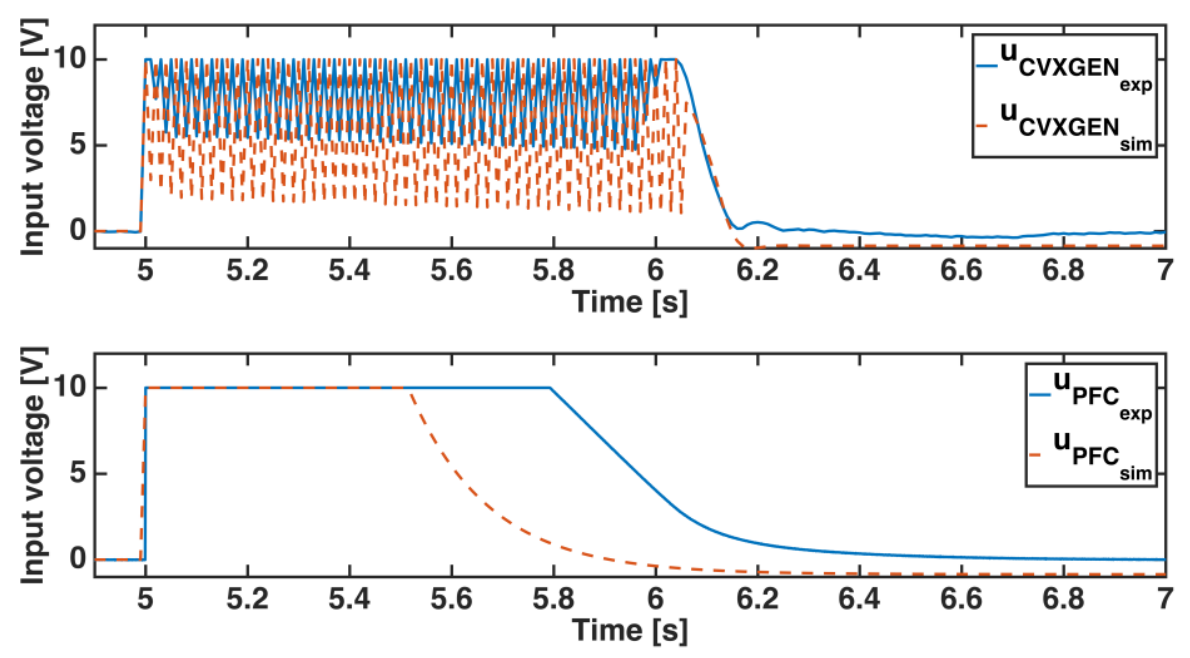

Fig. 4 Control signal of CVXGEN-MPC and PFC in simulation and experiment.

Figure 4 shows the different control signal given by control algorithms respectively. The control signal of CVXGEN-MPC is oscillating vigorously about the $10 \mathrm{~V}$ shows that the controller is trying to provide best control signal without violating the actuator slew rate constraint. On the other hand, PFC gives a smooth control signal, which is capped by the saturation point, at $10 \mathrm{~V}$. Stronger control signal is given in experiment than simulation is caused by the deviation in simulation plant model, which some of the actuator characteristics are not included.

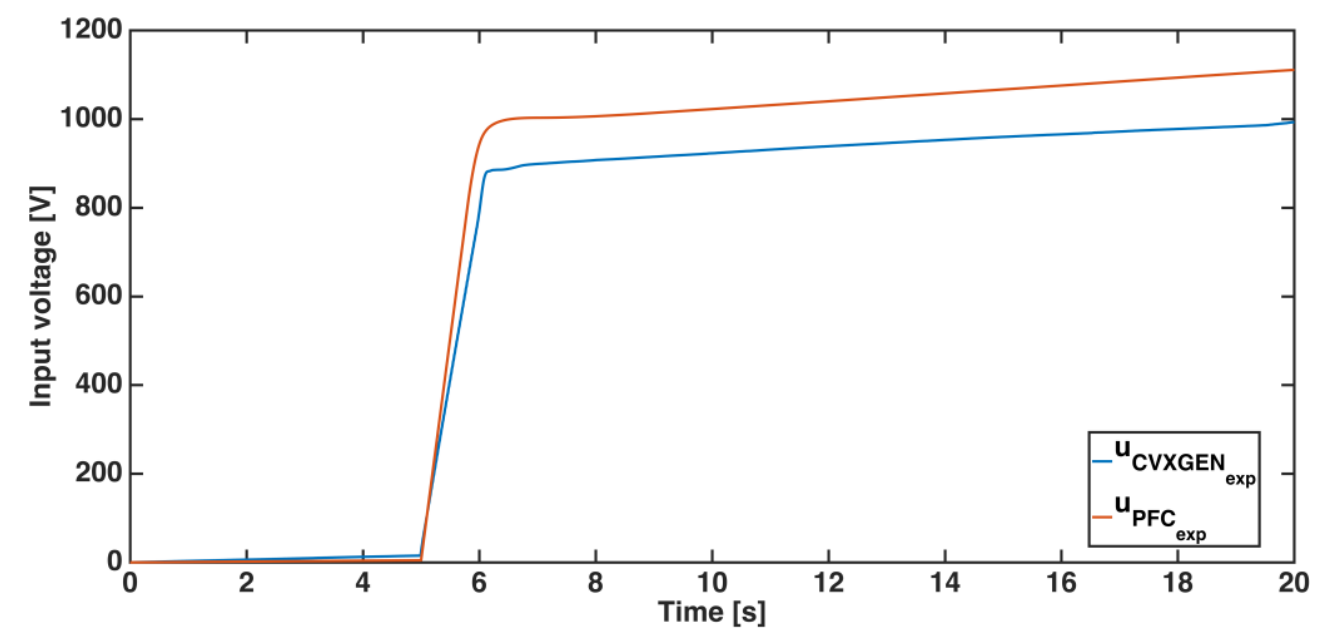

Fig. 5 Control effort of CVXGEN-MPC and PFC in experiment.

The control effort of the controller represents how efficient the controller to minimise the input changes while achieving the control objective at the same time. From the Figure 5, the sum of control input from PFC is $9.919 \%$ higher than one from CVXGEN-MPC.

Table 3 briefly summarised the control performance between CVXGEN-MPC and PFC. CVXGEN-MPC has an overall better performance over PFC in settling time, steady state error and control effort, even though PFC has a slightly better rise time over CVXGEN-MPC.

Table 3 - Experiment result comparison.

\begin{tabular}{cc}
\hline $\begin{array}{c}\text { Performance } \\
\text { Specifications }\end{array}$ & $\begin{array}{c}\text { CVXGEN-MPC } \\
\text { based on PFC }\end{array}$ \\
\hline Rise Time, $T_{r}$ & $-0.886 \%$ \\
Settling Time, $T_{s}$ & $2.538 \%$ \\
Steady State Error & $81.679 \%$ \\
Control Effort & $9.914 \%$ \\
\hline
\end{tabular}




\section{Conclusion}

In this paper, we presented the model development of EHA using system identification for obtaining dynamic characteristics of the system. Three types of input signals are used for the system identification method, and we identified model of EHA. Next, we proposed Model Predictive Control (MPC) with control signal optimization using CVXGEN. The proposed control algorithm is validated with Predictive Functional Control (PFC). Both algorithms are tested and evaluated for position control with and without disturbances in both Matlab simulation and real-time experimental on EHA. The collected results have shown the capability of CVXGEN-MPC, which is able to handle actuator constraint and better control performance with lower steady state error as compared to PFC. Future works of the system is to implement the EHA with the position control for tough robotic application such as actuator for legged robot or robotic arm.

\section{Acknowledgement}

This research is fully supported by UTM GUP grant, PY/2018/03018. The authors fully acknowledged Ministry of Higher Education (MOHE) and Universiti Teknologi Malaysia for the approved fund which makes this important research viable and effective.

\section{References}

[1] C. Morton, C. M. Spargo, and V. Pickert, "Electrified hydraulic power steering system in hybrid electric heavy trucks," IET Electr. Syst. Transp., vol. 4, no. 3, pp. 70-77, 2014.

[2] B. Li, J. Yan, G. Guo, H. Wang, and M. Zhang, "Identification of dynamic parameters and friction coefficients for a heavy-duty hydraulic manipulator," Proc. World Congr. Intell. Control Autom., no. 3, pp. 3102-3106, 2012.

[3] W. Li, J. Wang, F. Yang, and G. Zou, "An advanced selective hydraulic control system for the heavy-duty rough grinding machine,” Proc. IEEE Conf. Decis. Control, pp. 6046-6051, 2009.

[4] L. V Guo-fang, L. I. Zhong-hao, and J. I. Peng, "Design and Application of Hydraulic Synchronous Lift Control System Based on WebAccess,” pp. 5684-5686.

[5] T. Ljung, L. \& Glad, Modeling of Dynamic Systems. PTR Prentice Hall, 1994.

[6] S. Konami, Hydraulic Control Systems. Wiley, 2016.

[7] H. Yanada and K. Furuta, "Adaptive control of an electrohydraulic servo system utilizing online estimate of its natural frequency," Mechatronics, vol. 17, no. 6, pp. 337-343, 2007.

[8] N. Sepehri and G. Wu, "Experimental evaluation of generalized predictive control applied to a hydraulic actuator," Robotica, vol. 16, no. 4, pp. 463-474, 1998.

[9] H. M. Kim, S. H. Park, J. H. Song, and J. S. Kim, "Robust Position Control of Electro-Hydraulic Actuator Systems Using the Adaptive Back-Stepping Control Scheme,” J. Syst. Control Eng., vol. 224, no. 6, pp. 737-746, 2010.

[10] M. Choux, H. R. Karimi, G. Hovland, M. R. Hansen, M. Ottestad, and M. Blanke, "Robust Adaptive Backstepping Control Design for a Nonlinear Hydraulic-Mechanical System,” Jt. 48th IEEE Conf. Decis. Control 28th Chinese Control Conf., pp. 2460-2467, 2009.

[11] V. Lukanin, "Inverse kinematics, forward kinematics and working space determination of 3DOF parallel manipulator with S-P-R joint structure," Period. Polytech. Mech. Eng., vol. 49, no. 1, pp. 39-61, 2005.

[12] J. X. Xu, C. C. Hang, and C. Liu, "Parallel structure and tuning of a fuzzy PID controller," Automatica, vol. 36 , no. 5, pp. 673-684, 2000.

[13] N. J. M. P. B. M, "M041)Model-based pre edictive control applied to t a dual regenerative e and hydraulic brake sys stem," 2015.

[14] T. Tomatsu, K. Nonaka, K. Sekiguchi, and K. Suzuki, "Model predictive trajectory tracking control for hydraulic excavator on digging operation,” 2015 IEEE Conf. Control Appl. CCA 2015 - Proc., pp. 1136-1141, 2015.

[15]L. Sidhom, S. Sahraoui, and A. Abdelkrim, "Intelligent-PI controller for electro-hydraulic system," pp. 16-18, 2016.

[16] S. Sun, L. Dong, C. An, and W. Liu, "Fault-tolerant control design for linear systems with input constraints and actuator failures," 2009 Chinese Control Decis. Conf., pp. 5278-5283, 2009.

[17] G. Gattu and E. Zafiriou, "Nonlinear quadratic dynamic matrix control with state estimation," Ind. Eng. Chem. Res., vol. 31, no. 1986, pp. 1096-1104, 1992.

[18] R. Rouhani and R. K. Mehra, "Model algorithmic control (MAC); basic theoretical properties," Automatica, vol. 18, no. 4, pp. 401-414, 1982.

[19] R. R. Bitmead, M. Gevers, and V. Wertz, Adaptive Optimal Control: The Thinking Man's GPC. Prentice Hall New York, 1990.

[20] A. A. A. Emhemed, R. Bin Mamat, A. A. Mohd Faudzi, M. R. Johary, and K. Osman, "Modified predictive control for a class of electro-hydraulic actuator,” Int. J. Electr. Comput. Eng., vol. 6, no. 2, pp. 630-638, 2016. 
[21] N. H. Izzuddin, A. 'Athif M. Faudzi, M. R. Johary, and K. Osman, "System Identification and Predictive Functional Control for Electro-hydraulic Actuator System,” in 2015 IEEE International Symposium on Robotics and Intelligent Sensors (IRIS), 2015, vol. 16, no. June, pp. 138-143.

[22] I. Škrjanc, “A decomposed-model predictive functional control approach to air-vehicle pitch-angle control,” J. Intell. Robot. Syst. Theory Appl., vol. 48, no. 1, pp. 115-127, 2007.

[23] J. Mattingley, “Code Generation for Embedded Convex Optimization Convex optimization,” October, no. October, 2010.

[24] J. Mattingley and S. Boyd, "CVXGEN: A code generator for embedded convex optimization,” Optim. Eng., vol. 13, no. 1, pp. 1-27, 2012.

[25] A. A. M. Faudzi, N. H. I. Mat Lazim, and K. Suzumori, "Modeling and force control of thin soft McKibben actuator," Int. J. Autom. Technol., vol. 10, no. 4, pp. 487-493, 2016.

[26] R. Richalet and D. O’Donovan, Predictive Functional Control: Principles and Industrial Applications, vol. 53. Springer London, 2009.

[27] J. Y. Nagase, K. Hamada, T. Satoh, N. Saga, and K. Suzumori, "Comparison between PFC and PID control system for tendon-driven balloon actuator," IECON Proc. (Industrial Electron. Conf., no. 23760246, pp. 3398-3403, 2013.

[28] P. Poignet, "Predictive functional control for a parallel robot," Int. Conf. Intell. Robot. Syst., no. October, pp. 3-8, 2003.

[29] K. Osman, A. 'Athif M. Faudzi, M. F. Rahmat, O. F. Hikmat, and K. Suzumori, "Predictive Functional Control with Observer (PFC-O) Design and Loading Effects Performance for a Pneumatic System," Arab. J. Sci. Eng., vol. 40, no. 2, pp. 633-643, 2014.

[30] N. Bouton, R. Lenain, B. Thuilot, and P. Martinet, “An active anti-rollover device based on predictive functional control: Application to an all-terrain vehicle," in Proceedings - IEEE International Conference on Robotics and Automation, 2009, pp. 1309-1314.

[31] J. Rossiter and R. Haber, "The Effect of Coincidence Horizon on Predictive Functional Control," Processes, vol. 3, no. 1 , pp. 25-45, 2015.

[32] J. E. E. Bobrow and K. Lum, "Adaptive, high bandwidth control of a hydraulic actuator," in Journal of dynamic systems, measurement, and control, 1996, vol. 118, pp. 714-720.

[33] M. Hanger, T. A. Johansen, G. K. Mykland, and A. Skullestad, "Dynamic model predictive control allocation using CVXGEN," in Control and Automation (ICCA), 2011 9th IEEE International Conference on, 2011, pp. 417422.

[34] J. Mattingley, Y. Wang, and S. Boyd, “Code generation for receding horizon control," in Proceedings of the IEEE International Symposium on Computer-Aided Control System Design, 2010, pp. 985-992. 